computers. It was towards the end of the fiftes and at the beginning of the sixties that Alfred Niggli published with Hans Wondratschek a remarkable work on the generalization of symmetry groups or cryptosymmetry to quote them precisely. Their published works were at the source of a new domain in the field of mathematical crystallography. One can fully gauge their influence as these concepts are frequently used by present day researchers. V. A. Koptsik, author of many fundamental works on symmetry. took me in to his confidence and admitted that he considered Alfred Niggli as his master. An appreciation of this sort on the part of such an emminent author says very much for the significance of Alfred Niggli's work.

Niggli's knowledge of mathematical crystallography led him to close participation in the long elaboration of the new International Tables for Crystallography of which the first volume came out hardly a year ago. It must also be said that a good number of his works have remained unpublished, no doubt due to his own modesty. An example of this is a study on partially periodic space groups as well as a generalization of Hermann-Mauguin symbols applied to them.

I came to know and appreciate this generous personality as a PhD student in Alfred Niggli's research group. He was not just a crystallographer but also someone whose vast culture surpassed the title scientist. His knowledge of the classics led to him being called upon not only to appraise the Cantonal examinations in physics and chemistry but also in Latin. His knowledge of modern languages was equally impressive. What a surprise for Swedish guests on an official visit to Zürich to hear Alfred Niggli, then Rector of the University, addressing them in their own language.

Niggli often approached scientific problems with several well chosen words drawn from experiences during his journeys at home or abroad as well as from his activities in service for his country. He was also a music lover endowed with perfect pitch. He never forgot a theme and it is without doubt that this ability enabled him to take apart and analyse musical compositions. He discovered, particularly in Mozart's work, laws of symmetry similar to those one finds in one-dimensional freizes and borders. Some time ago he sent me a document showing some laws of symmetry in the Jupiter Symphony. It is today that I am able to estimate the significance of this last message from him. It is above all the radiance of his personality, his affability and his contributions to science that will leave the image of Alfred Niggli engraved in our memories.

\section{New Commercial Products}

Announcements of new commercial products are published by the Journal of Applied Crystallography free of charge. The descriptions, up to 300 words or the equivalent if a figure is included. should give the price and the manufacturer's full address. Full or partial inclusion is subject to the Editor's approval and to the space available. All correspondence should be sent to the Editor. Professor M. Schlenker. Editor Journal Applied Crystallography. Laboratoire Louis Neel du CNRS, BP166. F-38042 Grenoble CEDEX. France.

The International Union of Crystallography can as sume no responsibility for the accuracy of the claims made. A copy of the version sent to the printer is sen to the company concerned.

\section{J. Appl. Cryst. (1985). 18, 374-376}

IBM-PC-based Multichannel Digital Oscilloscope and Waveform Digitizer

LeCroy has recently introduced Waveform-Catalyst, a multichannel digital oscilloscope software package with powerful capabilities for acquisition, display, archiving, and analysis of high-speed waveforms. It is an integrated system consisting of LeCroy's series of modular waveform digitizers, any of the IBM personal computers (PC, Portable, XT or AT) and system software. Characteristics include:

Wideband Performance - Digitizer plugins offer bandwidths to $100 \mathrm{MHz}$ and maximum sampling rates of $200 \mathrm{MHz}$ or 5 nsec between points.

High Resolution Recording - The expandable waveform memory of up to $512 \mathrm{~K}$ points simplifies capturing signals with uncertain triggers, records signals of long duration, and provides the capability to zoom in on regions of particular interest.

Multichannel, Multi-timebase Operation - Up to 100 digitizers may be housed in benchtop or rackmounted mainframes, each with independent trigger, memory, and time/sample characteristics.

Modular Flexibility - The plug-in architecture permits selection of instrument characteristics, supports future upgrades, and protects against obsolescence. Friendly, Easy to Use - Catalyst operates like an instrument, not a computer. Single keystroke operating commands and familiar scope terminology make knowledge of computers or programming language unnecessary.

Powerful Display - The high resolution graphics permit simultaneous viewing of up to four "live" or previously stored traces, each with its own timebase and grid. Dual cursors control zoom, positioning, and the absolute differential measurement of time and voltage, while onscreen markers identify the trigger point, zero baseline, off-scale conditions, etc. Programmable Control, Archiving and Analysis - Any graph, instrument setup. or operations sequence can be stored and recalled from disk, or output to a graphics printer. And of course, there is the full power of the PC available for analysis, networking, or other computer operations.

Components for configuring an IBMPC-based waveform digitizing system are described in a new 10 page, colour brochure from LeCroy. LeCroy waveform digitizers consist of a family of mainframe housings into which plug-in digitizers, memories, signal conditioners and controllers are inserted to create an instrument that will meet general or application-specific signal measurement/analysis needs. A choice of three benchtop or rackmountable mainframes is offered, along with a family of high performance waveform digitizing modules. LeCroy's system components are described and illustrated with photos and selection charts for a variety of applications including waveform acquisition, storage, analysis, and display.

LeCroy Research Systems Ltd, Elms Court, Botley, Oxford OX2 9LP, England

\section{Computer-Controlled Guinier Diffrac- tometer System}

Huber Diffraktionstechnik GmbH has introduced a new line of computercontrolled Guinier-type diffractometers. Three different kinds of samples may be investigated: Flat powder specimens (Model 642), single capillary encapsulated powders or liquids (Model 644), and thin films or anodized layers on solid bulk materials (Model 653). The detector is either a $\mathrm{Na}(\mathrm{l})$ scintillation counter tube with a variable entrance slit or a straight position sensitive detector of $50 \mathrm{~mm}$ length. Both are positioned by a 500 steps/turn stepper motor. The resolution may be as low as $0.02^{\circ}(\theta)$, depending on sample conditions. There is evidently no distortion due to $\mathrm{K} \alpha_{2}$ or $K \beta$ radiation because of the focusing Johansson-type monochromator crystal. Together with a stepper motor control and a desktop computer, its graphically supported software is well suited for fast profile and phase analysis or intensity measurements for structure determination.

Models 644 and 653 may be equipped with a heater device for temperatures up to $1200 \mathrm{~K}$. For the thin-film instrument 653 , an additional rotation around the surface normal provides for the determination of preferred crystallite orientations or textures.

The software makes full use of CRT or matrix-printer graphics, featuring automatic search for diffraction peaks, precise refinement of angle position by a fitting procedure, smoothing of noisy scans, optimization of integration times and three-dimensional plots of diffrac- 
tograms. It runs on a HP personal computer and controls the hardware via RS232C or optional IEEE 488 interface.

Huber Diffraktionstechnik $\mathrm{GmbH}$, Sommerstrasse 4, D-8219 Rimstingl Chiemsee, Federal Republic of Germany

\section{Ductless Filtration Fume Cupboard}

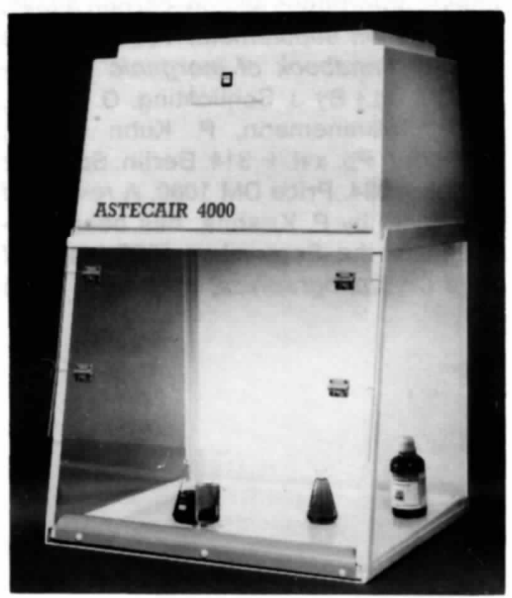

The Astecair-4000 fume cupboard

The new Astecair-4000 ductless filtration fume cupboard has been designed to fill a market need in combining the advantages of the large and/or multiple filters of the Astecair- 5000 with the smaller dimensions of the Astecair-3000. The unit has a glass reinforced plastic head unit and a strong steel framework with clear acrylic sides and a base measurement of $765 \times 665 \mathrm{~mm}$.

ASTEC also supply the range of Mystaire Scrubbing Systems, specially developed for the semi-conductor market, which are used for scrubbing exhaust gases from plasma etching and deposition systems as well as with MOCVD systems for the growth of crystals.

ASTEC Environmental Systems Ltd., 31 Lynx Crescent, Weston Industrial Estate, Weston-super-Mare, Avon BS24 9DJ

\section{Double-Crystal Topography Camera for Bent Samples - RTK2}

Compared to the conventional Lang topographical method, double-crystal topography offers the following advantages: shorter exposure times (more than one order of magnitude); higher resolution, higher strain sensitivity; layers with different lattice constants can be imaged separately, when working in the reflection mode (surface topography).
The camera RTK2 has the following properties: samples are in a horizontal position, no need for any kind of fixing (any glueing would cause additional strain!); the collimator can be bent automatically, in adaption to the curvature of a strained sample (minimum radius $5 \mathrm{~m}$ ); differences in lattice constants can be measured (resolution 10 $0^{-6}$ ); deformations can be measured quantitatively (down to $10^{-7}$ ); adjustment of sample and diffraction angle are performed semi-automatically; external computer can be used (option).

Complete topography measuring systems (including X-ray generator, detection system, process computer and software for controlling and analyzing) are also available.

EFG GmbH Elektrotechnische Fabrikations - U. Grosshandels GmbH, Düppelstrasse 13, D-1000 Berlin 37, Federal Republic of Germany

\section{New Generation of Philips Scanning Microscopes}

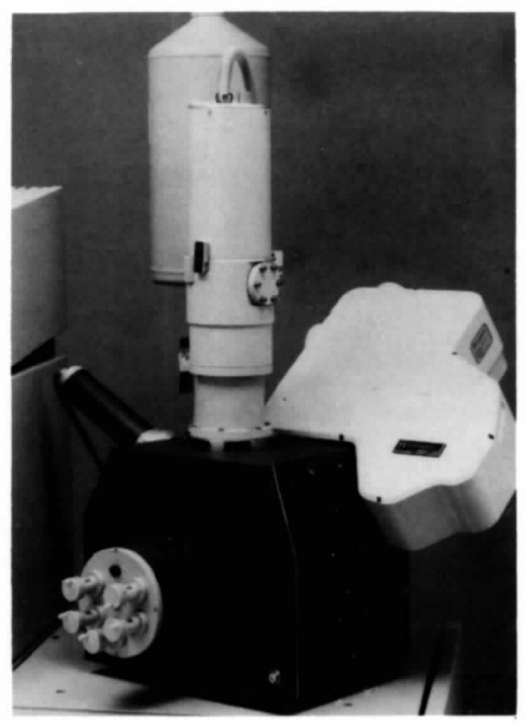

Philips SEM 535

Three new scanning electron microscopes, SEM 515, SEM 525 and SEM 535. will supersede the SEM 505 .

Higher resolution and a better signalto-noise ratio result from improvements in geometry within the unique Philips mini gun. In the special low-voltage configuration, with acceleration voltage reduced to 200 volts, brightness is increased, resulting in significantly better imaging of uncoated specimens. All instruments also offer the alternative high- brightness lanthanum hexaboride $\left(\mathrm{LaB}_{6}\right)$ emitter system.

There are three new pushbuttonselected functions. One is a pre-search or 'focus-finder' mode which brings the system into the general focus range. Once part of the image is visible, pushing a line-focus button then rapidly produces an accurate focus. The third button adjusts fine focus and astigmatism together. This new feature will shortly be available for retro-fitting to SEM $\mathbf{5 0 5}$ models also.

All instruments accept an improved version of the Philips multifunction detector system (MFD) in which four detectors with fibre-optic couplings are grouped round the specimen, providing TV-rate imaging of backscattered electrons and cathodoluminescence. Viewing systems can receive signals simultaneously from different detectors operating in the same or different modes, either combining them to produce an integrated image or comparing different modes in a split-screen display.

All three instruments can be interfaced to an external computer, with access to the data link for full computer control of microscope parameters.

SEM 515 is the general-purpose SEM that directly replaces the SEM 505. The universal goniometer stage provides more than 90 degrees tilt at the eucentric point without losing the feature of interest. Resolution of $5 \mathrm{~nm}$ at $30 \mathrm{kV}$ is guaranteed, with $4 \mathrm{~nm}$ easily obtainable. Both energy-dispersive (ED) and wavelength-dispersive (WD) $x$-ray analysis systems with an optimal take-off angle of 35 degrees can be fitted and operated simultaneously. ED and WD results may be combined for quantification.

SEM 525 is especially aimed at the general materials and metallurgical laboratories, especially the semiconductor industry, having an extremely large specimen stage. MFD and ED analysis systems can be used together, again employing a 35-degree take-off angle. Improved topographical imaging and magnetic contrast can also be obtained.

SEM 535 is particularly orientated to analytical work on large specimens. Its specially designed specimen chamber accepts very large samples for simultaneous analysis by ED and WD systems.

Pye Unicam Ltd, York Street, Cambridge CB1 2PX, England

\section{New Philips Software extends Role of Spectrometers}

Philips Analytical has introduced a new generation of software packages for its PW 1404 sequential and PW 1606 simulta- 
neous spectrometers, so that the spectrometers can now be used for a range of investigations not previously possible.

The use of a gamma correction, for example, is of particular interest to the steel industry and use of this term results in a considerable improvement in the accuracy of X-ray analysis of stainless steels. The advance is demonstrated in the analysis of austenitic stainless steels using a PW 1404 equipped with a scandium side window $X$-ray tube. For both $\mathrm{Cr}$ and $\mathrm{Ni}$, which are present in austenitic steels at relatively high concentrations (10-20 per cent), application of the gamma correction term, now available in the concentration-based $\mathrm{PH}$ model in Philips new generation software, results in accuracies better than $0.05 \%$.

Pye Unicam Ltd, York Street, Cambridge, CB1 2PX, England

\section{Semiconductor Application for Philips EDS Analyser}

Measurement of thin-layer films on silicon slices for the semiconductor industry is the application area for a second Philips Analytical PV 9500 energydispersive X-ray spectrometer delivered to the Mullard laboratory, Southampton.

With the latest software for coatings analysis, COAT 95 , the PV 9500 is aiding quality control procedures through the analysis of $\mathrm{P}_{2} \mathrm{O}_{5}$ glass on silicon.

Mullard's first PV 9500 was used for a completely different purpose - the analysis of cadmium mercury tellurium for use in infrared detectors. The two Mullard applications illustrate the many and diverse areas in which PV 9500s are proving valuable.

Pye Unicam Ltd, York Street, Cambridge, CB1 2PX, England

\section{Books Received}

J. Appl. Cryst. (1985). 18, 376

The following books have been received by the Editor. Brief and generally uncritical notices are given of works of marginal crystallographic interest: occasionworks of marginal crystallographic interest. Occasionthis heading because of difticu

Properties of crystalline silicon carbide. Diodes; molecular species in the gas phase; amorphous silicon-carbon alloys. (The silicon supplement, Vol. B2 of the Gmelin handbook of inorganic chemistry, 8th ed.) By J. Schlichting, G. Czack, E. Koch-Bienemann, P. Kuhn and F. Schröder. Pp. xvi + 314. Berlin: Springer Verlag, 1984. Price DM 1080. A review of this book, by $\mathrm{P}$. Krishna, has been published in the September 1985 issue of Acta Crystallographica, Section A, page 511. 